\title{
Performance of Variable Rate Bit Interleaved Coding for High Bandwidth Efficiency
}

\author{
Vincent K.N. Lau \\ knlau@eee.hku.hk \\ Department of Electrical and Electronic Engineering \\ University of Hong Kong
}

August 13, 1999

\begin{abstract}
In this paper, we propose a bandwidth efficient error correction scheme, namely the variable rate adaptive bitinterleaved coded modulation (ABICM), for wireless mobile channel. The code rate and modulation level are varied according to the current channel state to exploit the timevarying nature of the wireless channel. Design challenges to achieve symbol-by-symbol adaptation and component codes design are addressed. A multi-level puncturing scheme is proposed to solve the problem of symbol-by-symbol puncturing and interleaving. The optimal adaptation thresholds are derived. It is found that there are significant gains relative to the fixed rate coding in terms of SNR and throughput. It is also found that the ABICM scheme is essentially not degraded in small interleaving depths. This makes the ABICM very suitable for real time applications.
\end{abstract}

\section{Introduction}

One important bottleneck in wireless communications is to find bandwidth-efficient modulation and channel coding schemes to protect data over hostile mobile radio channels. The idea of combining channel modulation and coding, namely the trellis coded modulation (TCM), was purposed[1] to offer high bandwidth-efficient protection. It has been shown [2] that the order of diversity of any TCM in Rayleigh fading channel is the minimum number of distinct symbols ${ }^{1}$ between two codewords. Therefore, bitinterleaved coded modulation (BICM), which breaks a modulation symbol by bit-by-bit interleaving, has been purposed to increase the code-diversity order[3].

On the other hand, to better exploit the time varying nature of the channel, variable rate adaptive channel coding and modulation has been proposed [4]. The code rate and modulation level are varied according to the instantaneous channel condition. The receiver estimates the channel states and informs the transmitter through the use of a low capacity feedback link. When the channel state is good, the transmitter increases the instantaneous throughput by using a higher level modulation and higher code rate. On the other hand, when the channel state is bad, the trans-

\footnotetext{
${ }^{1}$ Minimum number of distinct symbols between any two codewords is called the minimum Hamming distance.
}

mitter uses a lower level modulation and lower code rate to maintain a targeted error level. In this way, a significant performance gain relative to fixed rate coding in terms of SNR and average throughput was obtained. In fact, this concept of variable rate adaptive coding and modulation has been widely applied in 3rd generation TDMA systems like Enhanced Data rate for GSM Evolution (EDGE) and UWC136.

It is therefore a natural extension to combine the above two concepts, namely the BICM and the variable rate adaptive channel coding, together to achieve a synergy. One very simple way to combine the two concepts is to adopt a multiframe adaptation technology similar to the EDGE system where the coding mode are fixed for a number of frames. Of course, performance gains are limited and it is not trival to extend to a symbol-by-symbol adaptation design Challenges remained to be solved include.

Puncturing and Interleaving: Symbol-by-symbol dynamic puncturing, together with interleaving, presents a design problem due to interleaving delay.

Optimal Adaptation Thresholds: It is not clear how the adaptation thresholds should be set especially in a symbol-by-symbol environment.

In this paper, we address the above questions. In section 2, we outline the design of the proposed ABICM and propose a new multi-level puncturing and interleaving scheme that could tackle the dilemma between interleaving and symbol-by-symbol puncturing. In section 3, we formulate the optimal adaptation thresholds for the ABICM. In section 4, we study the performance gain by simulations. In particular, we show that the symbol-by-symbol ABICM scheme proposed is very robust to bursty channel errors and this is very useful when large interleaving depth is not feasible for some real time applications. Finally, we conclude with a brief summary of results in section 5 .

\section{Adaptive Bit-Interleaved Coded Modulation}

The simplified system block diagram of the proposed scheme is shown in fig. 1(b). The transmitter consists of a variable rate punctured convolutional encoder, a bit-by-bit 
interleaver, a variable throughput adaptive modulator, and a channel state predictor. The variable rate convolutiona encoder instantaneouly varies the encoding rate by puncturing which periodically deletes coded bit streams based on some puncturing patterns. In the design, incremental punctured code is used. A family of QAM constellations (BPSK, QPSK, 8PSK, 16QAM, 32QAM, 64QAM) is used for the modulator with variable modulation throughputs. The available throughput modes are listed below.

Mode-0: rate-1/2 + 2 BPSK: throughput $=1 / 2$

Mode-1: rate-1/2 + 1 QPSK: throughput $=1$

Mode-2: rate-2/3 + 1 8PSK: throughput $=2$

Mode-3: rate-3/4 + 116QAM: throughput $=3$

Mode-4: rate-4/5 + 1 32QAM: throughput $=4$

Mode-5: rate-5/6 + 1 64QAM: throughput $=5$

Channel states are estimated at the receiver and fed back to the transmitter via a low capacity feedback link with certain delay. Appropriate encoding modes and modulation constellations are determined symbol-by-symbol at the transmitter based on the fed-back channel states. At the receiver, given the transmission mode sequence, a single Viterbi decoder could be used to determine the ML received sequence for all modes.

\subsection{Multi-level Puncturing and Interleav- ing}

Interleaving is used to convert the bursty fading into independent fading. Puncturing is used to achieve variable rate encoding. Conventionally, puncturing is followed by interleaving and followed by modulation. However, for variable rate adaptive coding system with symbol-by-symbol adaptation, dynamic puncturing is a problem. This is because puncturing modes have different puncturing periods which usually span several symbol durations. If there are a number of mode changes within a period, rate-compatibility or incremental puncturing pattern is difficult to maintain.

Furthermore, when interleaving is considered as well, the puncturing and the interleaving tasks are not trival. When coded bits enters the interleaver, the puncturing level for the bits (which is determined by the channel states when the bits are actually transmitted) has to be fixed. However, there is always a huge interleaving delay from the time bits enter the interleaver and the time the bits are actually taken out of the interleaver and transmitted. Therefore, puncturing could not be done before interleaving. On the other hand, puncturing could neither be done after interleaving. Otherwise, the designed puncturing pattern on the trellis diagram could not be maintained because it is possible that bits out of the interleaver actually correspond to non-puncturable bit positions in the original trellis diagram.

The solution to the problems is by multilevel puncturing and interleaving. We label the coded bits that are puncturable and defer the puncturing action after interleaving. Instead of a single block interleaver, we have a number of parallel interleavers of different levels. This is illustrated in figure 1 and [5]. Level- 0 interleaver contains coded bits that are non-puncturable. Level-1 interleaver contains coded bits that are puncturable according to puncturing pattern-1. Bits from level- 0 interleaver must be transmitted by modulation symbols while bits from the other levels of interleavers are puncturable and therefore, could be transmitted or not transmitted (punctured).

With this layered approach, puncturing could be done after interleavers. This is illustrated in figure 1(a). For example, if 8PSK is chosen, 3 coded bits are taken from the level-0 interleaver and one puncturable bits are taken from the level-1 interleaver. The 3 coded bits are used to modulate a 8PSK symbol while the puncturable bit is not transmitted.

\subsection{Operation of the ABICM}

Transmission mode $m$ is chosen if the predicted CSI at the transmitter, $\hat{z}$, lies between $\left[\zeta_{m}, \zeta_{m+1}\right] .\left\{\zeta_{m}\right\}$ are the adaptation thresholds.

We have two different ways to operate the ABICM scheme, namely constant BER operation and constant throughput operation[4].

Constant BER Operation: The adaptation thresholds are set to maintain a relatively constant BER over a range of average SNR. Average throughput of the $A B I C M$ is compared relative to the fixed rate code at a given BER and at the same SNR.

Constant Throughput Operation: The adaptation thresholds are set to maintain a constant average throughput relative to the average SNR. SNR of the ABICM is compared relative to the fixed rate codes at a given BER and at the same throughput.

\subsection{Mode Synchronization}

In ABICM, transmitted symbols have a varying modulation modes and puncturing levels depending on the instantaneous channel states. At the receiver, correct decoding relies on the knowledge of the transmission mode sequence and this refers to mode synchronization between the transmitter and the receiver. Mode synchronization can be attained with a closed loop method by embedding control words on the transmitted frame[4].

\section{System Analysis}

\subsection{Decision Metric}

Let $\vec{c}=\left[c_{1}, c_{2}, \ldots, c_{N}\right]$ be the transmitted coded bit sequence and $\vec{s}=\left[s_{1}, s_{2}, \ldots, s_{N}\right]$ be the associated label position sequence for the coded bits. The ML decoding metric for the $s$-th bit of the label of the transmitted symbol $x$ is , given by:

$p\left(\mathbf{y} \mid \rho, m, l^{\circ}(\mathbf{x})=b\right)=$
$\quad \sum_{\mathbf{x} \in \mathcal{X}(m)} p(\mathbf{y} \mid \rho, \mathbf{x}) P\left(\mathbf{x} \mid l^{\prime}(\mathbf{x})=b\right)=\frac{1}{2^{g(m)-1}} \sum_{\mathbf{x} \in \mathcal{X}_{b}^{i}(m)} p(\mathbf{y} \mid \rho,(\mathbf{z})$ 
where $\rho$ is the channel fading, $g(m)$ is the constellation size for mode- $m, \mathcal{X}_{b}^{i}(m)$ is the set of constellation points with the $i$-th bit given by $b$.

\subsection{Optimal Adaptation Thresholds}

For the constant throughput operation, the problem is to choose the adaptation thresholds, $\left\{\zeta_{i}\right\}$ so as to minimize the BER with the constraint of constant average throughput. On the other hand, for the constant BER operation, the problem is to choose the thresholds so as to maximize the average throughput with the constraint of constant BER with respect to the SNR. It is shown in [5] that the optimal thresholds for both operation modes have the same form given by:

$$
\begin{aligned}
& {\left[\beta\left(\zeta_{1}, 1\right)-\beta\left(\zeta_{1}, 0\right)\right]=\left[\beta\left(\zeta_{2}, 2\right)-\beta\left(\zeta_{2}, 1\right)\right]} \\
& \quad=\cdots=\left[\beta\left(\zeta_{M-1}, M-1\right)-\beta\left(\zeta_{M-1}, M-2\right)\right]
\end{aligned}
$$

\section{Results and Discussion}

To reduce the simulation time, a core rate $1 / 2$ code with constraint length $K=5$ is used. Due to the variable throughput in the ABICM scheme, we use the average symbol energy to noise ratio, $\frac{E_{2}}{\eta_{0}}$, instead of the usual bit energy to noise ratio as a reference for comparison. We assumed the forward baud rate is $500 \mathrm{k}$ baud with the symbol duration $T_{s}=2 \mu s$. The maximum mobile speed of interest is $100 \mathrm{~km} / \mathrm{hr}$ and the corresponding Doppler frequency $f_{d} \approx 200 \mathrm{~Hz}$ at $2 \mathrm{GHz}$ band. The required feedback channel capacity is $7.2 \mathrm{kbps}$ which is much lower than the forward capacity.

The ABICM system is operated in constant throughput and constant BER operations. The performance in terms of SNR gain and throughput gain is compared with the corresponding BICM schemes. Note that the SNR gains or the throughput gains are two sides of the same coin. It depends on the adaptation control to realize either one or both types of gains simultaneously.

\subsection{Ideal Performance of ABICM}

In this section, we consider the ideal performance of the ABICM scheme. Ideal refers to perfect interleaving and zero feedback delay.

Constant Throughput Operation: The BER performance of the ABICM system relative to the fixedrate BICM system is plotted in figure 2. Note that along the BER curves of the ABICM scheme, the average throughput remains constant. Therefore, the relative SNR gains could be compared with the corresponding fixed rate BICM codes at the same throughput. For example, there are $4 \mathrm{~dB}, 5 \mathrm{~dB}, 5 \mathrm{~dB}$ SNR gains of the ABICM schemes relative to the 8PSK$\operatorname{BICM}(\bar{\gamma}=2), 16 \mathrm{QAM}-\mathrm{BICM}(\bar{\gamma}=3), 32 \mathrm{QAM}-\mathrm{BICM}$ $(\bar{\gamma}=4)$ schemes respectively at BER level of $10^{-4}$.

Constant BER Operation: Figure 3 shows the BER curves of the ABICM schemes operating in the constant $B E R$ mode. The BER levels off over a range of SNRs called the adaptation range. Outside the range, the SNR is either too large or too small and the ABICM is essentially operating on mode- 6 or mode- 0 respectively. Note that along the BER curves of the ABICM schemes, the average throughput is varying as illustrated in figure 3(b). To compare the performance with fixed-rate BICM schemes, we have to look at the throughput gains at the same BER and SNR points. For example, there are throughput gains of around 1.5 times at $\mathrm{BER}=10^{-4}, 10^{-5}$ and $10^{-6}$ respectively.

\subsection{Effects of Block Interleaving}

Figure 4 (a) and (b) illustrates the BER curves (at constant average throughput) of ABICM and BICM schemes with various interleaving depths at $f_{d} T_{s}=6 \times 10^{-3}$. Note that fixed-rate BICM schemes are degraded significantly relative to the ideal interleaving situation at all throughputs. On the other hand, the ABICM schemes are much more robust to bursty channel errors. For example, the SNR gains from figure 4 become $10 \mathrm{~dB}, 11 \mathrm{~dB}$ relative to the 8PSK-BICM $(\bar{\gamma}=2), 16 \mathrm{QAM}-\mathrm{BICM}(\bar{\gamma}=3)$ respectively at BER $=10^{-4}$ and interleaving depth of $64 \times 64$. Therefore, symbol-bysymbol adaptive channel coding schemes are good choices for protecting data over bursty channels when large-depth interleaving is not feasible for realtime applications.

\subsection{Effect of Feedback Delay and Noisy Feedback}

Estimated channel states are fed back to the transmitter with certain delay of $\Delta$ symbol durations. Assume the symbols on the feedback channel are transmitted with SNR $E_{f b} / \eta_{0}$. Figure 5(a) and (b) shows the degradations of ABICM with respect to $\Delta$ and $E_{f b} / \eta_{0}$. Since there is no interleaving in the feedback codewords, the required $E_{f b} / \eta_{0}$ for reasonable performance is quite large (about $20 \mathrm{~dB}$ ). However, since the baud rate on the feedback channel is much smaller than the forward channel (70 times or $18 \mathrm{~dB})$, the actual required transmitted power for the feedback codewords is not high at all.

\section{Conclusions}

In this paper, we propose a variable rate symbol-by-symbol adaptive error correction scheme error correction scheme, namely the ABICM, to exploit the time varying nature of the mobile radio channel for high bandwidth-efficiency applications. A low capacity feedback channel is used to convey channel state information to the transmitter. Design issues such as the symbol-by-symbol puncturing and interleaving, the schemes to maintain mode synchronization, the formulation of optimal adaptation thresholds, the effect of feedback delay, noisy feedback and finite interleaving depth are addressed. For the first time, we introduce multi-level puncturing to achieve symbol-by-symbol adaptation. It is found that there are significant SNR gains (up to 5dB) and throughput gains (up to 1.5 times) of the ABICM scheme. The scheme is also shown to be robust to bursty channel errors and feedback delay. Therefore, the scheme is very 


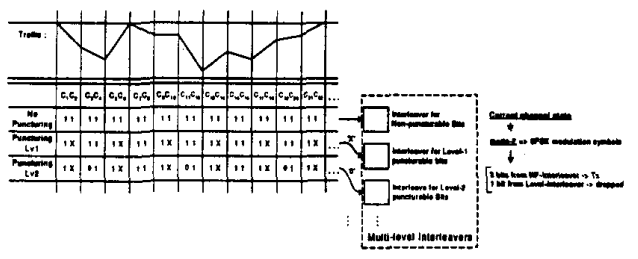

(a) Multi-level Puncturing

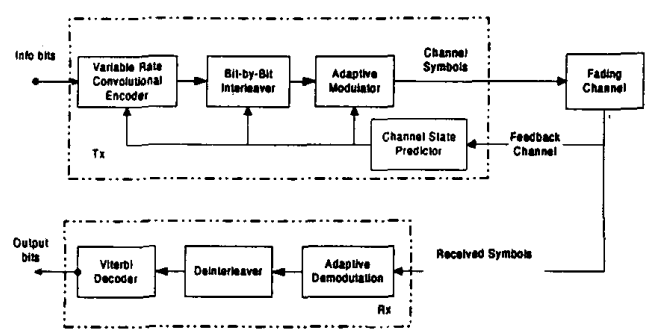

(b) Overall Block Diagrams of the ABICM Scheme.

Figure 1: Multi-level puncturing and system block diagram.

useful especially in cases where large interleaving depth is not be feasible.

\section{References}

[1] G. Ungerboeck, "Channel Coding with multilevel/phase signals," IEEE Trans. on Info. Theory, vol. IT-28, pp. 55-67, Jan. 1982.

[2] D. Divsalar and M. K. Simon, "Trellis Coded Modulation for 4800-9600 bits/s Transmission Over a Fading Mobile Satellite Channel," IEEE JSAC., vol. SAC-5, pp. 162-175, Feb. 1987.

[3] X. Li and J. A. Ritcey, "Trellis Coded Modulation with Bit Interleaving and Iterative Decoding," IEEE JSAC., vol. 17, pp. 715-724, April 1999.

[4] K. N. Lau, "Channel Capacity and Error Exponent of Variable Rate Adaptive Channel Coding for Rayleigh Fading Channel," To appear in IEEE Trans. on Communs.

[5] K. N. Lau, "On the design of Variable Rate, Symbol-bySymbol, Adaptive Bit Interleaved Trellis Coded Modulation.," Submitted to IEEE JSAC., 1999.

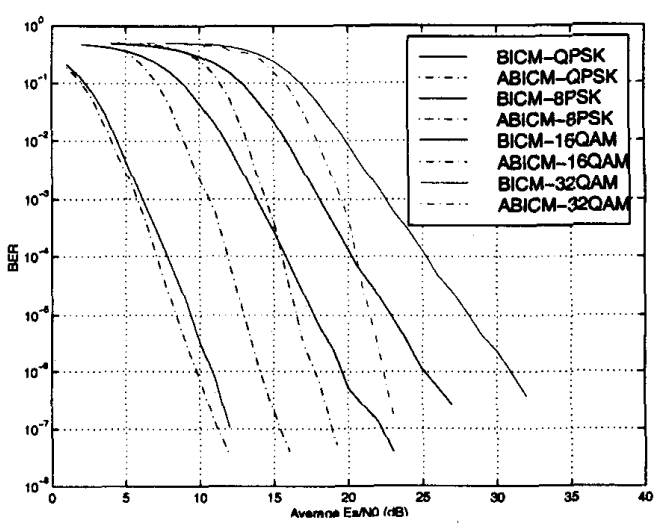

Figure 2: Performance of ABICM at constant throughput control.

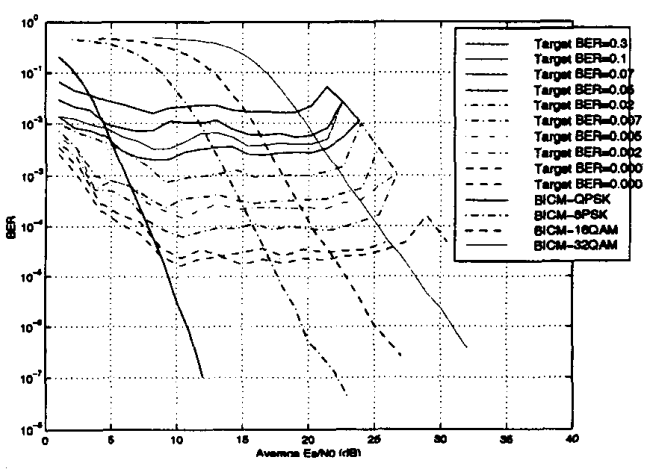

(a) BER against SNR at constant BER control

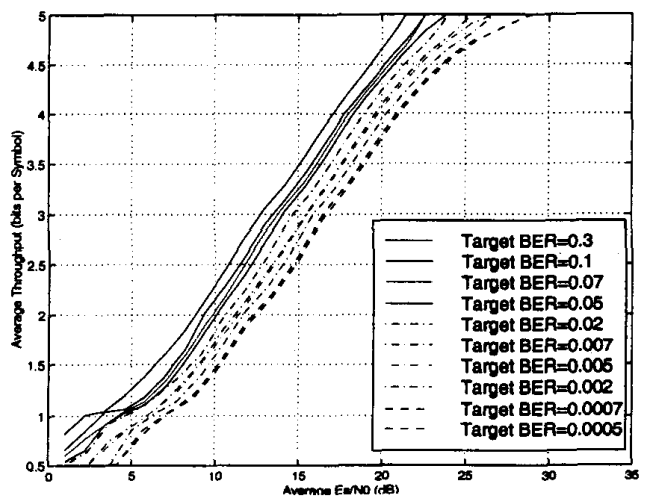

(b) Throughput against SNR at constant BER control

Figure 3: Performance of ABICM at constant BER control. 


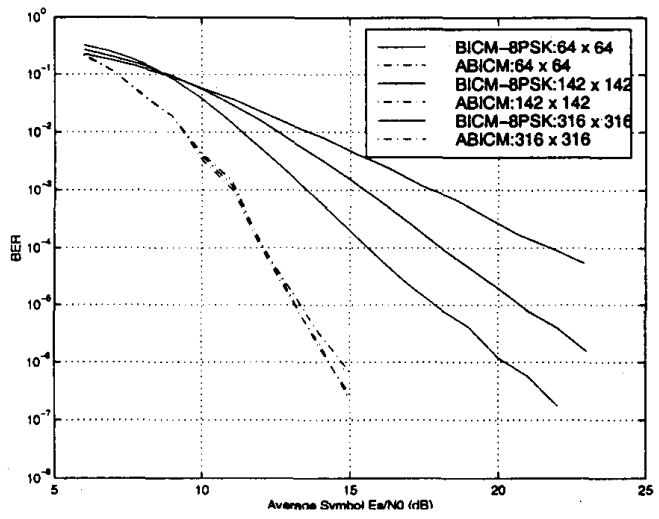

(a) BER against SNR for ABICM-8PSK

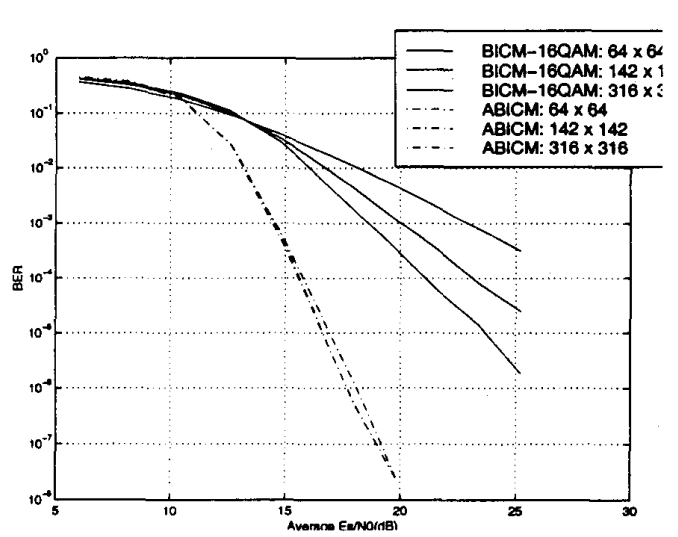

(b) BER against SNR for ABICM-16QAM

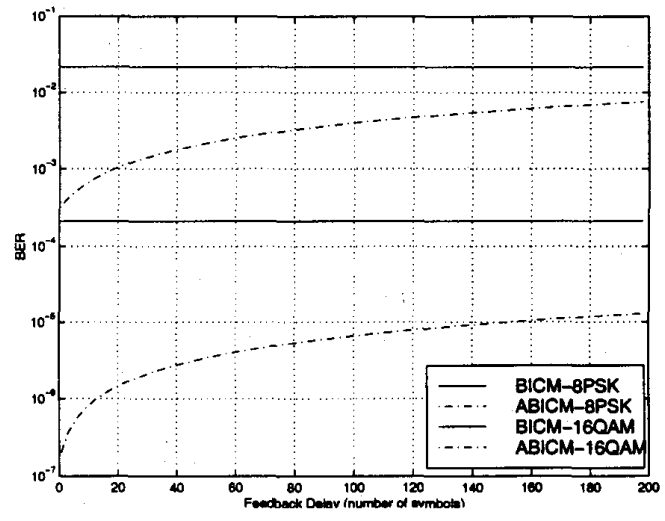

(a) Effect of Feedback Delay

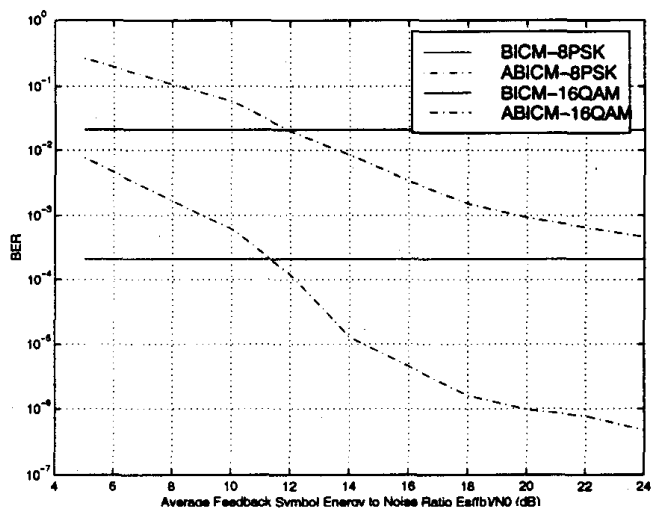

(b) Effect of Feedback Noise

Figure 5: Robustness of ABICM with noisy and delayed

Figure 4: Performance of ABICM at constant throughputs. 